\title{
Closed-Loop Handling Stability of 4WS Vehicle with Yaw Rate Control
}

\author{
Hongming $\mathrm{Lv}^{*}$ - Shaona Liu \\ Yancheng Institute of Technology, School of Automotive Engineering, China
}

\begin{abstract}
To improve the vehicle handling stability, a four-wheel steering (4WS) controller with yaw rate tracking is proposed in this paper. A driver/ vehicle closed-loop system is built based on the preview-follower theory to evaluate the system's manoeuvring performance. Next, the system driving on a double lane-change road and on a low adhesion road are simulated and compared with conventional front-wheel steering vehicle. The Fourier transform method is developed to analyse the dynamics of the closed-loop system in the frequency domain. The results show that the proposed 4WS vehicle has the preferred response characteristics of sideslip angle and yaw rate, and is robust regarding tire cornering stiffness, which guarantees the handling stability in emergency braking condition.
\end{abstract}

Keywords: four-wheel steering, vehicle, handling stability, closed-loop system, driver, yaw rate

\section{INTRODUCTION}

With the rapid development of global transportation, the amount of traffic accidents has been increasing year by year. Consequently, many countries have enhanced the development of automotive active safety systems. For the potential of improving vehicle handling stability, the 4WS (Four-Wheel Steering) system has attracted much attention from companies and researchers over the previous 40 years [1] and [2]. Although 4WS is one of the current focuses of vehicle dynamics control and many researchers have presented various opinions on the configuration and control strategy, automotive companies still tend to adopt the active rear steering configuration, which is simple, economic and reliable [3] to [5].

For a $4 \mathrm{WS}$ vehicle, the conventional zero sideslip control strategy [6] can help driver to have the correct feeling of the vehicle body movement when vehicle corners, but it decreases the steady-state gain of the vehicle yaw rate a lot at high speed, and thus leads the vehicle to excessive understeering in that it is impossible to decouple the two variables of sideslip angle and yaw rate completely with only one control variable of the rear wheel steering angle [7].

Due to the above mentioned reasons, some researchers began to incorporate $4 \mathrm{WS}$ into the chassis integrated control system with direct yaw-moment control (DYC) or an active suspension system (ASS) and so on [8] to [10]. Some others took the optimising the control objective in order to improve the vehicle handling performance by only using 4WS technology, which not only considered the coordination of the sideslip angle and yaw rate, but also the nonlinearity of tire and the variation of road condition [11] to [14].
In the 1970s, Sorgatz and Weir proposed a driver model to build a driver/vehicle closed-loop system to handle the problem of evaluating the handling characteristics of designed vehicle [15] and [16]. However, for most 4WS control methodologies, the emphases were put on the vehicle dynamics models and 4WS controller logic themselves, i.e. the analysis and synthesis only considered the independent system including the vehicle model and 4WS controller. Thus, there have only been a few studies taking into account the human effect of the driver in the controller design and synthesis of 4WS [17] and [18].

The objective of this paper is to investigate the design of a $4 \mathrm{WS}$ controller to reduce the dynamic errors of yaw rate and sideslip angle by tracking the proposed vehicle model, and to evaluate the closedloop system performance including driver and the 4WS vehicle. The paper is organised as follows. In Section 1, a 2DOF (degree-of-freedom) lateral dynamics model of 4WS vehicle is built. A control algorithm with yaw rate tracking is proposed in Section 2. In Section 3, the closed-loop system of driver and 4WS vehicle is built. The handling characteristics of the driver/vehicle system on a double lane-change road and limited road conditions are simulated and compared to those of a front-wheel steering (FWS) vehicle. A frequency analysis method is proposed to evaluate the handling performance of the vehicle/driver closed system. Finally, some conclusions are drawn in Section 4.

\section{LATERAL DYNAMICS OF 4WS VEHICLE}

A vehicle is a very complex system, so many of parameters are usually required if a multi-body dynamics method is adopted to model it in detail. To 
minimise the complexity and difficulty of developing a vehicle dynamics control system, it is common to build a relatively simple vehicle model and thus validate the feasibility in the concept design stage.

In order to study the essential vehicle dynamics and simplify the analysis procedure, the influence of roll on lateral motion is assumed to be small and not taken into account in this paper. A 2DOF model containing sideslip and yaw rate, commonly used in vehicle dynamics control, is utilised to study the handling stability of $4 \mathrm{WS}$ vehicle, as shown in Fig. 1 [13] and [19].

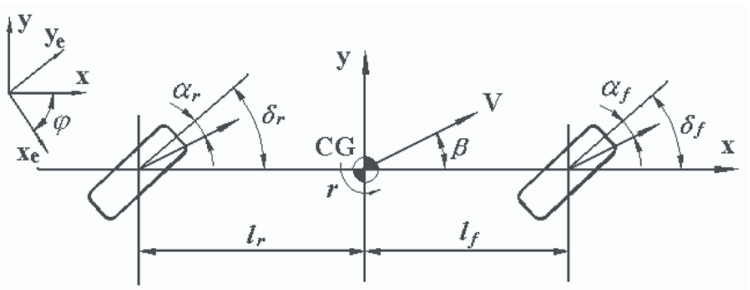

Fig. 1. 2DOF vehicle lateral dynamic model

The inertial coordinate system, $\left(x_{e}, y_{e}, z_{e}\right)$ is fixed on the ground, where the $z_{e}$ axis represents the direction normal to the $\left(x_{e}, y_{e}\right)$ plane. This serves as a reference frame for vehicle motions. The body (chassis) coordinate system, denoted by $(x, y, z)$ with its origin at the $C G$, is introduced to describe vehicle motion. Next, the chassis system, $(x, y, z)$ is rotated a yaw angle $\varphi$ with respect to the inertial system $\left(x_{e}, y_{e}\right.$, $z_{e}$ ).

In Fig. $1, \beta$ and $r$ denote the sideslip angle and yaw rate of vehicle at the CG; $m$ is the vehicle mass; $J_{z}$ is the yaw moment of inertia about its mass centre $z$-axis; $V$ denotes the velocity of vehicle; $l_{f}$ and $l_{r}$ are the distances from the CG (centre of gravity) to the front and rear axles; $\delta_{f}$ and $\delta_{r}$ denote the steering angles of front and rear wheels; $\alpha_{f}$ and $\alpha_{r}$ are the slip angles of front and rear tires; $C_{f}$ and $C_{r}$ denote the lateral stiffnesses of the front and rear tires, respectively.

Only considering lateral and yaw motions, the vehicle dynamic equations can be derived by applying Newton's second law.

Lateral motion:

$$
m V(\dot{\beta}+r)=F_{f} \cos \delta_{f}+F_{r} \cos \delta_{r} .
$$

Yaw motion, or moments about the vertical z-axis through the $\mathrm{CG}$ :

$$
J_{Z} \dot{r}=l_{f} F_{f} \cos \delta_{f}-l_{r} F_{r} \cos \delta_{r} .
$$

In general, lateral tire force is a non-linear function of the slip angle. In this study, the cornering stiffness for the front (rear) wheel is denoted by $C_{f}\left(C_{r}\right)$ and its value depends on the tire-road interaction. As long as the tire slip angle is small, a linear relationship between tire force and slip angle can be justified. Then, the lateral forces generated by the front and rear tires vary linearly with their slip angles.

$$
F_{f}=C_{f} \alpha_{f} \text { and } F_{r}=C_{r} \alpha_{r} .
$$

If the sideslip angle $\beta$ is small and vehicle velocity $V$ varies slowly, $\alpha_{f}$ and $\alpha_{r}$ will be given by:

$$
\alpha_{f}=\delta_{f}-\beta-\frac{l_{f}}{V} r \quad \text { and } \quad \alpha_{r}=\delta_{r}-\beta+\frac{l_{r}}{V} r .
$$

In addition, $\delta_{f}$ and $\delta_{r}$ are generally small,

$$
\cos \delta_{f} \approx 1 \text { and } \cos \delta_{r} \approx 1 \text {. }
$$

Hence, the vehicle motion equations are expressed by

$$
\left\{\begin{array}{l}
m V(\dot{\beta}+r)=-2\left(C_{f}+C_{r}\right) \beta-\frac{2\left(l_{f} C_{f}-l_{r} C_{r}\right)}{V} r \\
+2 C_{f} \delta_{f}+2 C_{r} \delta_{r} \\
J_{Z} \dot{r}=-2\left(l_{f} C_{f}-l_{r} C_{r}\right) \beta-\frac{2\left(l_{f}^{2} C_{f}+l_{r}^{2} C_{r}\right)}{V} r \\
+2 l_{f} C_{f} \delta_{f}-2 l_{r} C_{r} \delta_{r}
\end{array} .\right.
$$

Define the state vector $x=\left[\begin{array}{ll}\beta & r\end{array}\right]^{T}$, input vector $u=\left[\begin{array}{ll}\delta_{f} & \delta_{r}\end{array}\right]^{T}$ and output vector $y=\left[\begin{array}{ll}\beta & r\end{array}\right]^{T}$, the vehicle model can be written in the state-space form as follows:

$$
\begin{aligned}
& \dot{x}=A x+B \delta_{f}+E \delta_{r} \\
& y=C x
\end{aligned}
$$

where, the coefficient matrices are:

$$
\begin{gathered}
A=\left[\begin{array}{cc}
-\frac{2\left(C_{f}+C_{r}\right)}{m V} & -\frac{2\left(l_{f} C_{f}-l_{r} C_{r}\right)}{m V^{2}}-1 \\
-\frac{2\left(l_{f} C_{f}-l_{r} C_{r}\right)}{J_{Z}} & -\frac{2\left(l_{f}^{2} C_{f}+l_{r}^{2} C_{r}\right)}{J_{Z} V}
\end{array}\right], \\
B=\left[\begin{array}{l}
\frac{2 C_{f}}{m V} \\
\frac{2 C_{f}}{J_{Z}}
\end{array}\right], C=\left[\begin{array}{ll}
1 & 0 \\
0 & 1
\end{array}\right], E=\left[\begin{array}{c}
\frac{2 C_{r}}{m V} \\
-\frac{2 C_{r}}{J_{Z}}
\end{array}\right] .
\end{gathered}
$$




\section{4WS CONTROLLER}

One of the main advantages of a 4WS vehicle is that there are more choices to realise the desired handling performance. Proportional control based on steady zero sideslip can improve the vehicle manoeuvrability at low speeds [20]. However, the control strategy may significantly decrease the yaw rate gain in that only one control input is used in the 2DOF system, which is not suitable for the handling of vehicle at high speed. The proposed control objective in this paper is to reduce both the harmonic peak values of yaw rate and sideslip angle as much as possible [13], viz. to improve the vehicle handling stability at high speed while avoid excessive understeer, an optimal controller for $4 \mathrm{WS}$ vehicle based on yaw rate tracking is designed as shown in Fig. 2.

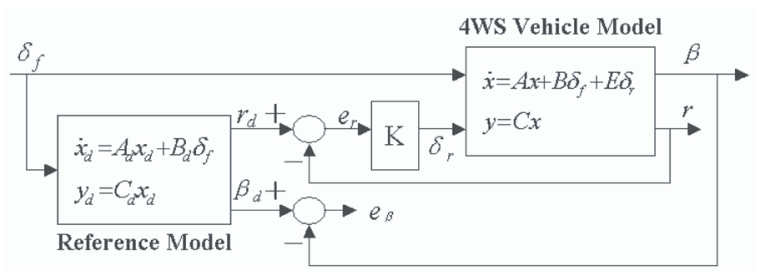

Fig. 2. 4WS control system

The desired reference yaw rate and sideslip angle are first order time lag transfer functions of the steering angle of front wheel, i.e.

$$
\begin{aligned}
& r_{d}=\frac{r_{0}}{1+\tau_{r} s}, \\
& \beta_{d}=\frac{\beta_{0}}{1+\tau_{\beta} s},
\end{aligned}
$$

where, $\tau_{r}$ and $\tau_{\beta}$ are the time constants of yaw rate and sideslip angle respectively; $r_{0}$ is the steadystate gains of yaw rate of FWS vehicle, and $\beta_{0}$ is the corresponding steady-state gains of sideslip angle. They are derived from Eq. (6).

$$
\begin{gathered}
\left.r_{0}=\frac{r}{\delta_{f}}\right)_{\text {steady }}=\frac{V /\left(l_{f}+l_{r}\right)}{1+\frac{m}{l_{f}+l_{r}}\left(\frac{l_{f}}{2 C_{r}}-\frac{l_{r}}{2 C_{f}}\right) V^{2}}, \\
\left.\beta_{0}=\frac{\beta}{\delta_{f}}\right)_{\text {steady }}=\frac{1-\frac{m l_{f}}{2\left(l_{f}+l_{r}\right) l_{r} C_{r}} V^{2}}{1+\frac{m}{l_{f}+l_{r}}\left(\frac{l_{f}}{2 C_{r}}-\frac{l_{r}}{2 C_{f}}\right) V^{2}} \frac{l_{r}}{\left(l_{f}+l_{r}\right)} .
\end{gathered}
$$

The state-space representation of the desired reference vehicle model is given by:

$$
\left\{\begin{array}{l}
\dot{x}_{d}=A_{d} x_{d}+B_{d} \delta_{f} \\
y_{d}=C_{d} x_{d}
\end{array}\right.
$$

where the state vector $x_{d}$ and output vector $y_{d}$ are written as follows, $x_{d}=\left[\begin{array}{ll}\beta_{d} & r_{d}\end{array}\right]^{T}$ and $y_{d}=\left[\begin{array}{ll}\beta_{d} & r_{d}\end{array}\right]^{T}$.

And the coefficient matrices are:

$$
\begin{gathered}
A_{d}=\left[\begin{array}{ll}
A_{d 1} & A_{d 2}
\end{array}\right]=\left[\begin{array}{cc}
-\frac{1}{\tau_{\beta}} & 0 \\
0 & -\frac{1}{\tau_{r}}
\end{array}\right], \\
B_{d}=\left[\begin{array}{l}
B_{d 1} \\
B_{d 2}
\end{array}\right]=\left[\begin{array}{l}
\beta_{0} \\
r_{0}
\end{array}\right], \quad C_{d}=\left[\begin{array}{ll}
1 & 0 \\
0 & 1
\end{array}\right] .
\end{gathered}
$$

The physical interpretation of this control algorithm is that when the front wheel is steered, the yaw rate is measured and compared with an ideal yaw rate response $r_{d}$. Next, the rear wheel steering angle is controlled by controller $K$.

$$
\delta_{r}=K \cdot\left(r_{d}-r\right) .
$$

In order to make the outputs of an actual vehicle to follow the outputs of desired model, the error variables are defined as $e_{\beta}=\beta_{d}-\beta, e_{r}=r_{d}-r$ while the state vector $\hat{x}=\left[\begin{array}{llll}\beta & r & e_{\beta} & e_{r}\end{array}\right]^{T}$ and output vector $\hat{y}=\left[\begin{array}{ll}e_{\beta} & e_{r}\end{array}\right]^{T}$. Note that only $e_{r}$ is used to control the system directly, and $e_{\beta}$ is used to optimise the controller in the design stage. The equivalent state equations of $4 \mathrm{WS}$ vehicle including the controller $K$ are obtained from Eqs. (7), (12) and (13).

$$
\left\{\begin{array}{l}
\hat{\dot{x}}=\hat{A} \hat{x}+\hat{B} \delta_{f} \\
\hat{y}=\hat{C} \hat{x}
\end{array},\right.
$$

where

$$
\begin{gathered}
\hat{A}=\left[\begin{array}{ccc}
A & 0 & K \cdot E \\
A_{d}-A & A_{d 1} & A_{d 2}-K \cdot E
\end{array}\right], \\
\widehat{B}=\left[\begin{array}{c}
B \\
B_{d}-B
\end{array}\right], \hat{C}=\left[\begin{array}{llll}
0 & 0 & 1 & 0 \\
0 & 0 & 0 & 1
\end{array}\right] .
\end{gathered}
$$

For the above standard output feedback problem, the controller $K$ can be designed according to optimal control theory or the $\mathrm{H} \infty$ control theory, etc. 
To minimise all the harmonic peak values of vehicle yaw rate and sideslip angle while not decreasing the steady-state response gain of yaw rate, a $\mathrm{H} \infty$ optimal controller is designed. The mathematic expression is described as,

$$
\min J=:\|W S\|_{\infty},
$$

where the weight $W=\left[\begin{array}{ll}w_{\beta} & w_{r}\end{array}\right] ; S$ is the sensitivity function matrix of $\beta$ and $r$ to $\delta_{f}$.

$$
S=\left[\begin{array}{ll}
e_{\beta} & e_{r}
\end{array}\right]^{T}=\widehat{C}(s I-\hat{A})^{-1} \widehat{B} .
$$

It is noted that the weight $W$ is mainly dependent on the experience and experiment. However, it is also found that the values of $w_{\beta}$ and $w_{r}$ are related closely to $e_{\beta}$ and $e_{r}$ in that the sensitivities of them to controller $K$ are quite different.

\section{CLOSED-LOOP HANDLING STABILITY OF 4WS VEHICLE}

\subsection{Modelling of Closed-Loop System including Driver and Vehicle}

Modelling the driver/vehicle closed-loop system has been a subject of research since the 1970s [15] and [16]. The driver's use of visual information in controlling the direction of a vehicle is obviously very significant and has been investigated extensively. There is a general consensus that control occurs at two levels [21] and [22]: preview control (openloop feedforward), in which the driver anticipates the path ahead and makes an appropriate steering action based on knowledge of the vehicle dynamics; and compensatory control (closed-loop feedback), in which the driver compensates for errors in the preview control and for disturbances. The compensatory task involves the human operator controlling a system to minimise an error.

Guo presented an algorithm to perform the modelling of the closed-loop driver/vehicle directional control system from a mathematical point of view, i.e. preview follower theory, which can be used to determine the main frame and all parameters of the system. The driver/vehicle closed-loop system mode is built as shown in Fig. 3. $T_{p}$ denotes the driver's lead time constant; $C_{0}$ represents the driver's compensatory gain, and equals to the reciprocal of the steady-state lateral acceleration gain; $T_{c}$ is the driver's correction time constant; $t_{d}$ is the driver's cognitive time delay; $T_{h}$ is a first-order lag time constant which represents the driver's neuromuscular time delay [23].

Ashkens and McRuer stated that the driver's delay time constants of $t_{d}$ and $T_{h}$ have nothing to do with the characteristics of vehicles and usually take the values about $t_{d}=0.2 \mathrm{~s}$ and $T_{h}=0.1 \mathrm{~s}$ while $C_{0}$, $T_{p}$, and $T_{c}$ depend on the vehicle characteristics, and driver experience [24].

On the basis of the previous investigation [23], Guo promoted the further work of driver model parameter identification by utilising a car driver simulator and presented the parameter's reference range [25]: $T_{p} \in[0.58,2.072], T_{c} \in[0.02,2.76]$, $t_{d} \in[0.17,0.53], T_{h} \in[0.08,0.36]$.

The vehicle model in Fig. 3 is rebuilt by defining

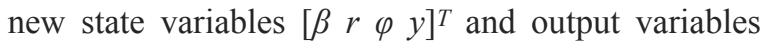
$\left[\begin{array}{lll}\beta & & y\end{array}\right]^{T}$ while $\delta_{f}$ and $\delta_{r}$ are control variables. The yaw rate $r$ is the differential of yaw angle $\varphi$.

$$
r=\dot{\varphi} .
$$

According to the vehicle dynamic characteristic shown in Fig. 1, the vehicle lateral velocity $\dot{y}$ can be deduced as,

$$
\dot{y}=V \sin (\beta+\varphi) \approx V(\beta+\varphi) .
$$

The new matrixes $A, B, C$ and $E$ of state equations are rewritten as following,

$$
A=\left[\begin{array}{cccc}
-\frac{2\left(C_{f}+C_{r}\right)}{m V} & -\frac{2\left(l_{f} C_{f}-l_{r} C_{r}\right)}{m V^{2}}-1 & 0 & 0 \\
-\frac{2\left(l_{f} C_{f}-l_{r} C_{r}\right)}{J_{Z}} & -\frac{2\left(l_{f}^{2} C_{f}+l_{r}^{2} C_{r}\right)}{J_{Z} V} & 0 & 0 \\
0 & 1 & 0 & 0 \\
V & 0 & V & 0
\end{array}\right],
$$

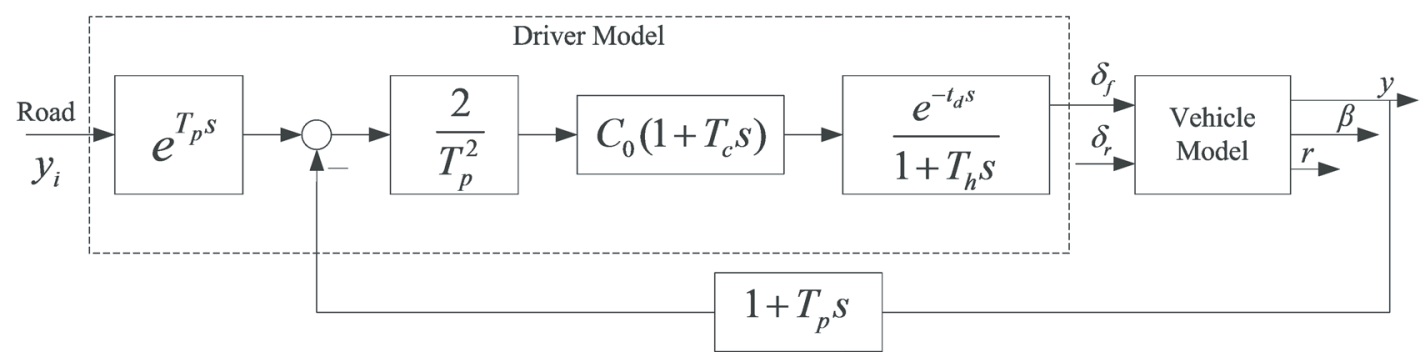

Fig. 3. Closed-loop system including driver and vehicle 


$$
B=\left[\begin{array}{c}
\frac{2 C_{f}}{m V} \\
\frac{2 C_{f}}{J_{Z}} \\
0 \\
0
\end{array}\right], C=\left[\begin{array}{llll}
1 & 0 & 0 & 0 \\
0 & 1 & 0 & 0 \\
0 & 0 & 0 & 1
\end{array}\right], E=\left[\begin{array}{c}
\frac{2 C_{r}}{m V} \\
-\frac{2 C_{r}}{J_{Z}} \\
0 \\
0
\end{array}\right] .
$$

The test parameters of a Buick LeSabre, a fullsize sedan, are used to build the vehicle model [26] while the driver handling parameter ranges presented by Guo [23] and [25] are referred and synthesised to build the driver model. The key parameters of driver/ vehicle system are summarised in Table 1.

Table 1. Parameters of the driver/vehicle system

\begin{tabular}{lc}
\hline Parameter & Value \\
\hline Vehicle mass $(m)$ & $1740 \mathrm{~kg}$ \\
\hline Yaw inertia $\left(J_{Z}\right)$ & $3214 \mathrm{~kg} \cdot \mathrm{m}^{2}$ \\
\hline Front axle to CG $\left(I_{f}\right)$ & $1.058 \mathrm{~m}$ \\
\hline Rear axle to CG $\left(I_{r}\right)$ & $1.756 \mathrm{~m}$ \\
\hline Front tire cornering stiffness $\left(C_{f}\right)$ & $29000 \mathrm{~N} / \mathrm{rad}$ \\
\hline Rear tire cornering stiffness $\left(C_{r}\right)$ & $60000 \mathrm{~N} / \mathrm{rad}$ \\
\hline Reference yaw rate time lag $\left(\tau_{r}\right)$ & $0.1 \mathrm{~s}$ \\
\hline Reference sideslip angle time lag $\left(\tau_{\beta}\right)$ & $0.1 \mathrm{~s}$ \\
\hline Driver's lead time $\left(T_{p}\right)$ & $1 \mathrm{~s}$ \\
\hline Driver's compensatory gain $\left(C_{0}\right)$ & $1.7 \mathrm{~s} 2 / \mathrm{m}$ \\
\hline Driver's correction time $\left(T_{c}\right)$ & $0.2 \mathrm{~s}$ \\
\hline Driver's cognitive time delay $\left(t_{d}\right)$ & $0.28 \mathrm{~s}$ \\
\hline Driver's neuromuscular time delay $\left(T_{h}\right)$ & $0.1 \mathrm{~s}$ \\
\hline
\end{tabular}

\subsection{Simulation of Double Lane-Change Manoeuvre}

A double lane-change manoeuvre is a typical driving condition and commonly used to estimate vehicle handling performance. In this paper, it is adopted to simulate the lateral dynamics for both the 4WS and FWS vehicles at a speed of $30 \mathrm{~m} / \mathrm{s}$, see Fig. 4. The behaviour of front wheel steering angles of them during the manoeuvring process is given as shown in Fig. 5.

The trajectories of both vehicles are similar to the actual steering process, which proves the validity of the simulation. The steering angles of them are also very similar, which shows the simulation results of FWS and 4WS vehicles have comparability because they are at the same input condition.

Their sideslip angle and yaw rate responses on a double lane-change road are compared in Fig. 6. When a 4WS vehicle is cornering, the maximum

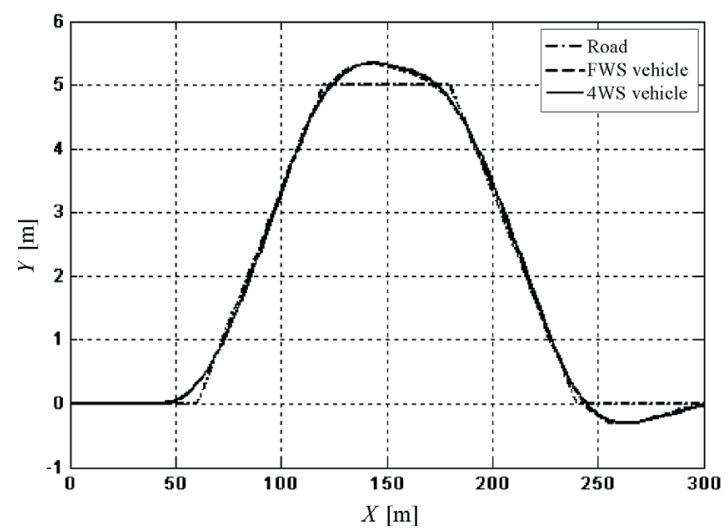

Fig. 4. Trajectories of road, 4 WS and FWS vehicle motion

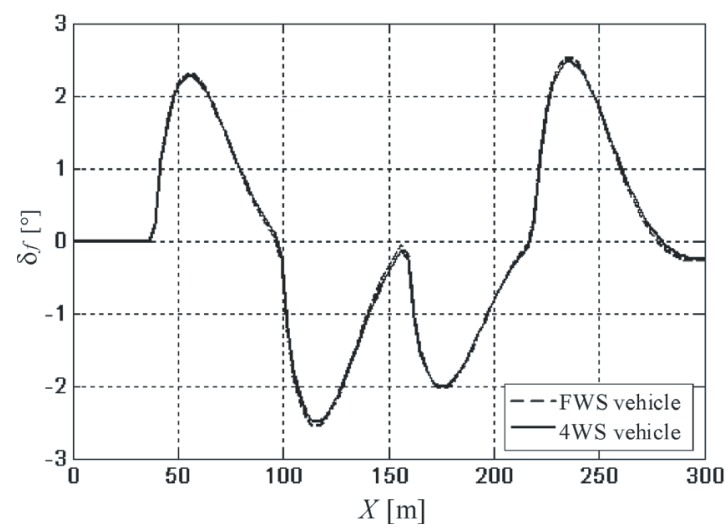

Fig. 5. Steering angles of front wheel

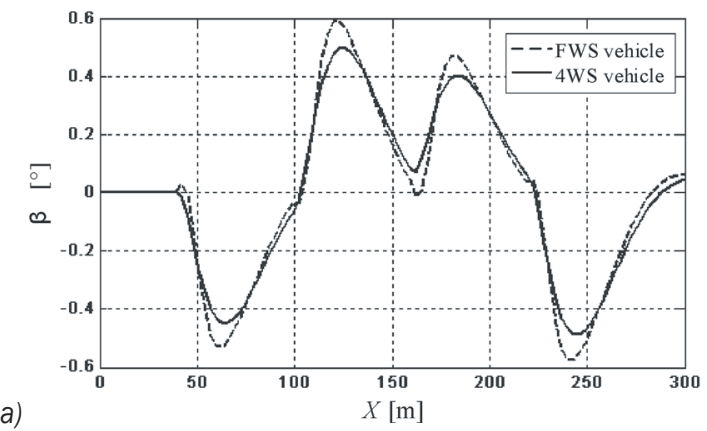

a)

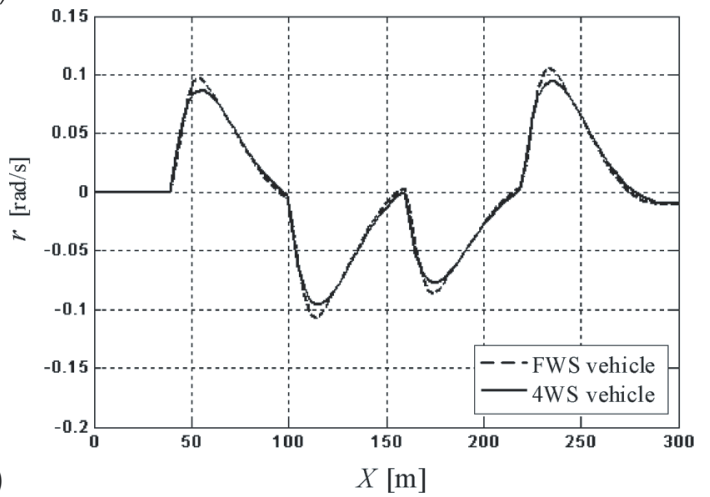

Fig. 6. Responses of vehicle state variables on double lanechange road; a) Sideslip angle, b) Yaw rate 
sideslip angle, compared to FWS vehicle, is obviously reduced while the maximum yaw rate is decreased to a certain extent.

The relationships of $\beta$ and $r$ to $\delta_{f}$ of both $4 \mathrm{WS}$ and FWS vehicles at a speed of $30 \mathrm{~m} / \mathrm{s}$ are compared in Fig.7.

The response curves of $4 \mathrm{WS}$ and FWS are similar to each other, but the maximum values of sideslip angle and yaw rate of $4 \mathrm{WS}$ vehicle are smaller than those of FWS vehicle, as the same in Fig. 6. Therefore, the time responses indicate that $4 \mathrm{WS}$ can improve the vehicle's dynamic handling behaviour to make the vehicle easier to drive at high speeds.

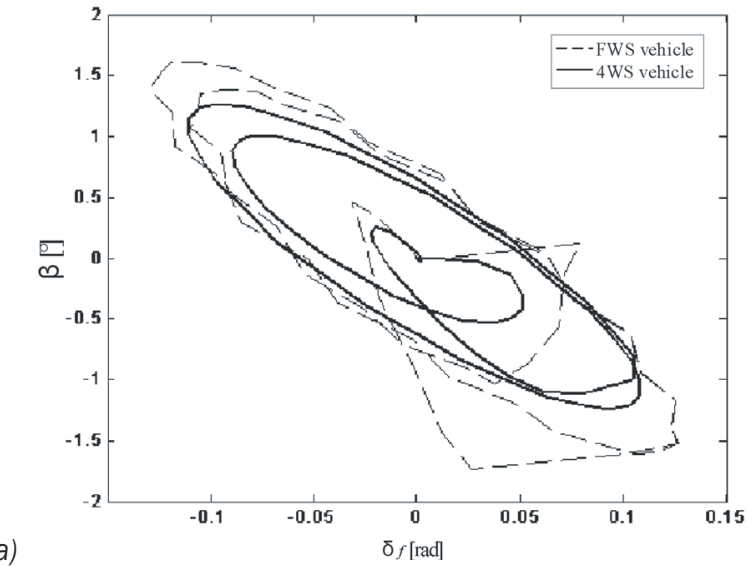

a)

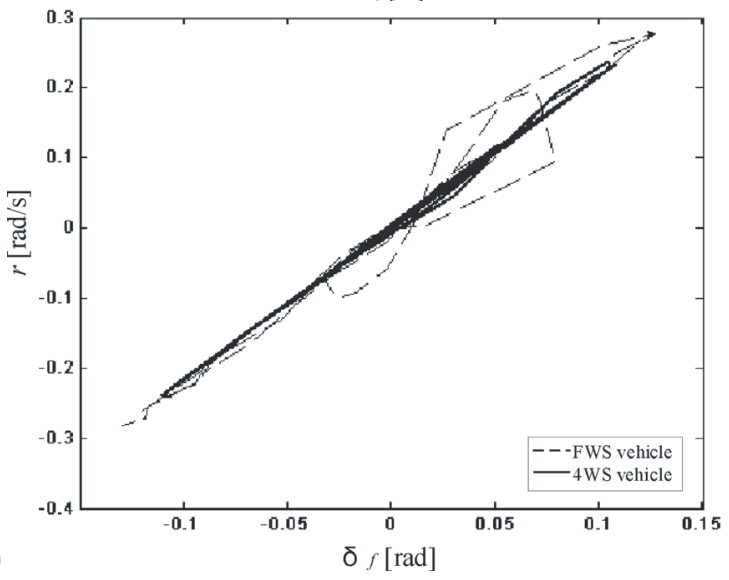

Fig. 7. Relationship of $\beta$ and $r$ to $\delta_{f}$ on double lane-change road; a) $\left.\beta-\delta_{f}, b\right) r-\delta_{f}$

\subsection{Analysis of Closed-Loop System in the Frequency Domain}

Though a double lane-change road is usually described its length in space, it can also be converted by time if the road length is divided by vehicle velocity. Thus, for a closed-loop system including driver and vehicle, the road is the time input signal while $\beta$ and $r$ are the outputs. It is known that the system transfer function is so complex that it cannot be written in a simple format. There is a direct way to obtain the system transfer function: the Fourier Transform method is used to the input and output variables in the simulation.

$$
H(j \omega)=\frac{Y(\omega)}{X(\omega)}=\frac{F[y(t)]}{F[x(t)]}=\frac{\int_{0}^{\infty} y(t) e^{-j \omega t} d t}{\int_{0}^{\infty} x(t) e^{-j \omega t} d t} .
$$

Here, $\mathrm{H}(j \omega)$ is the system transform function; $X(\omega)$ and $Y(\omega)$ are the input and output expressed by frequency; $x(t)$ and $y(t)$ are the input and output by time, respectively. Based on the above theory, the closed-loop dynamic characteristics in the frequency domain can be obtained by FFT (Fast Fourier Transform).

The dynamic characteristics of closed-loop system in frequency domain are depicted in Figs. 8 and 9.

The figures show that the resonances of driver/ vehicle closed-loop system mainly lie in the middle frequency range between 1 20 rad/s. The important natural frequencies, caused by vehicle and driver characteristics, are $1.5,3,4.5,6.3,8$ and $9.4 \mathrm{rad} / \mathrm{s}$, individually. They also indicate that the proposed 4WS controller is equivalent to a mid-pass filter for the driver/ vehicle closed-loop system. Compared to the FWS vehicle, the sideslip angle resonance peak values of the $4 \mathrm{WS}$ vehicle are decreased distinctly. The second resonance peak value is decreased is from 0.88 to $0.7 \mathrm{deg}$, the third is from 0.85 to $0.55 \mathrm{deg}$, and the fourth decreases especially, from 1.1 to $0.57 \mathrm{deg}$, respectively.

The yaw rate resonance peak values of $4 \mathrm{WS}$ vehicle are also decreased. The third peak value is decreased from 0.17 to $0.15 \mathrm{rad} / \mathrm{s}$ while the fourth is from 0.25 to $0.18 \mathrm{rad} / \mathrm{s}$.

In fact, the closed-loop handling stability evaluation is still developing and not effectively applied for the variation of driver's subjective properties. It is meaningful to evaluate the handling stability of a developing vehicle by utilising the resonance frequency analysis method to the driver/ vehicle closed-loop system.

\subsection{Simulation of Emergency Braking Condition}

Many traffic accidents occur in condition of emergency braking, which lead to the vertical loads of front and rear tires changing greatly. According to the lateral properties of tires, the equivalent 


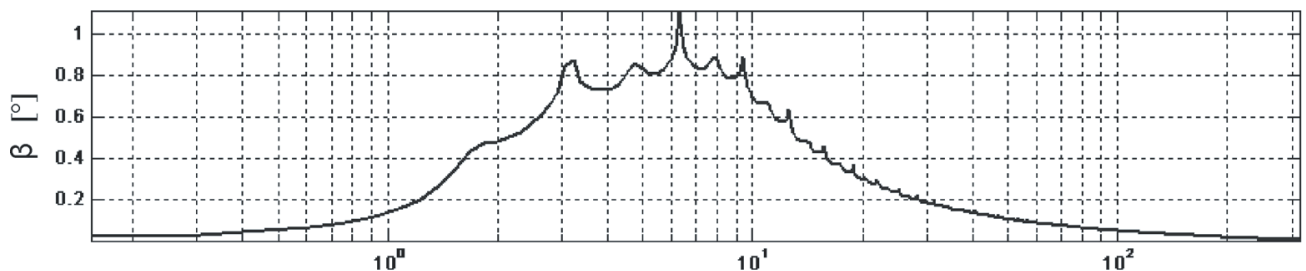

a)

Frequency [rad/s]

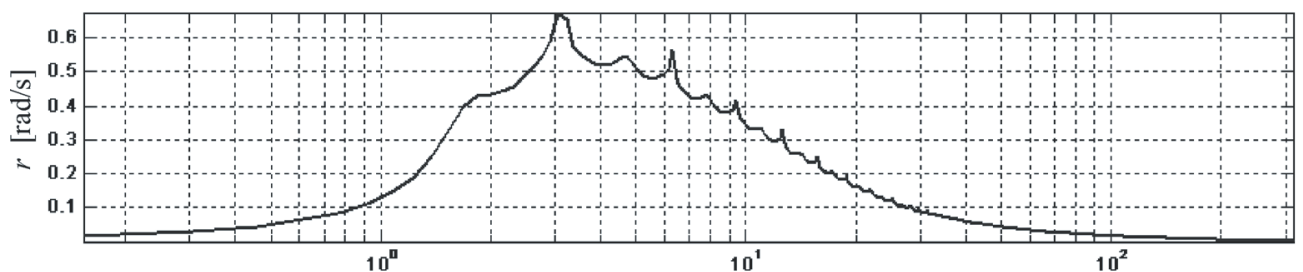

b)

Frequency [rad/s]

Fig. 8. Closed-loop dynamic characteristics of sideslip angle; a) FWS vehicle, b) 4WS vehicle

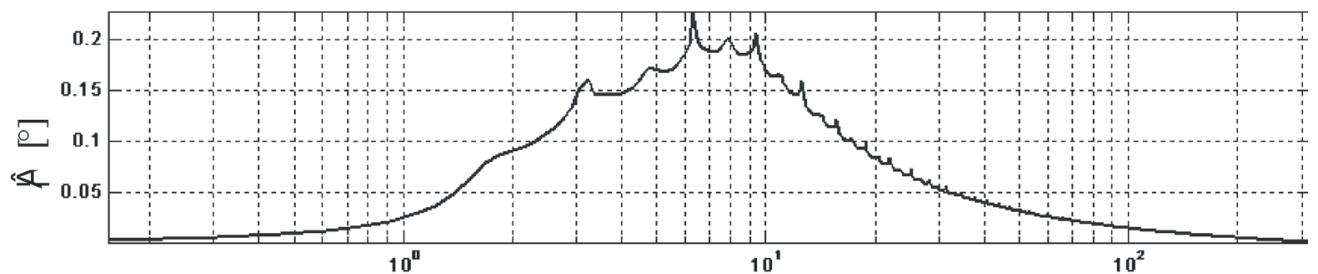

a)

Frequency $[\mathrm{rad} / \mathrm{s}]$

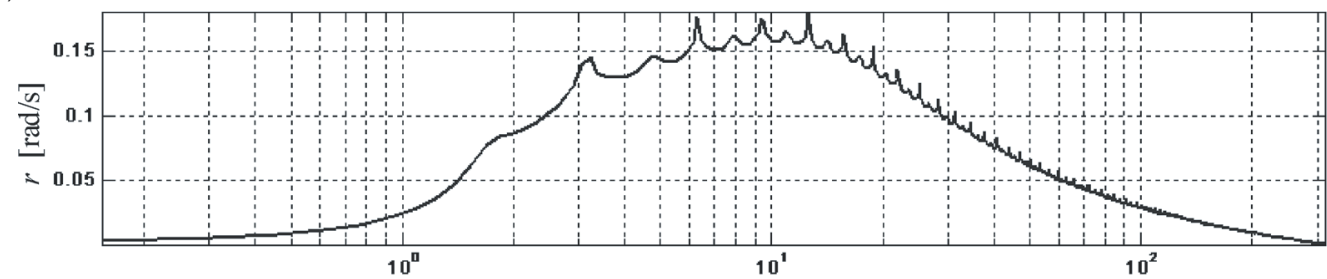

b)

Frequency $[\mathrm{rad} / \mathrm{s}]$

Fig. 9. Closed-loop dynamic characteristics of yaw rate; a) FWS vehicle, b) 4WS vehicle
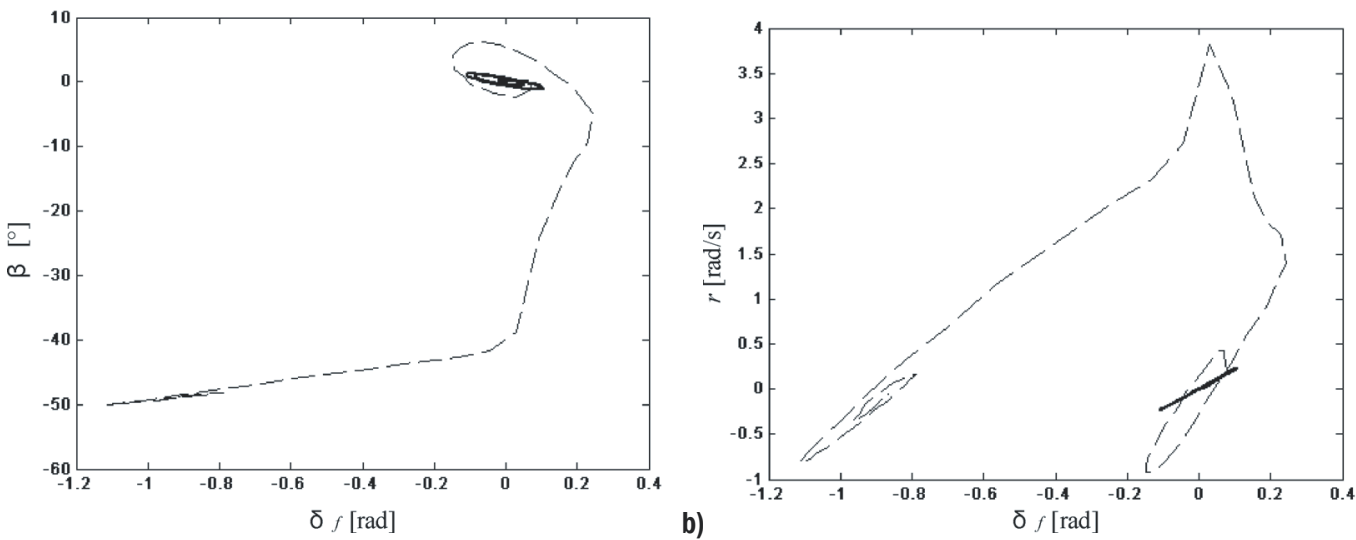

Fig. 10. Relationship of $\beta$ and $r$ to $\delta_{f}$ in emergency braking condition; a) $\left.\beta-\delta_{f}, b\right) r-\delta_{f}$

cornering stiffness of the front tire increases while the rear decreases, which reduces the vehicle's natural frequency and increases the response peak value of each state variable. Thus the handling stability of the vehicle is deteriorated, and the vehicle may be out of control. 
To simplify the study, it is assumed that the cornering stiffness of rear tire decreases by $50 \%$ [8]. The closed-loop systems of 4WS and FWS vehicles on a double lane-change road are simulated and the relationships of state variables are shown in Fig. 10.

When both types of vehicle are steered in such emergency braking condition, all the responses of FWS vehicle exceed the stable region while the 4WS vehicle still has the original characteristics, which shows that the 4WS vehicle is robust to the variation of tire cornering stiffness, and thus guarantees preferable handling stability.

\section{CONCLUSIONS}

According to the lateral dynamics simulation of a driver/vehicle closed-loop system on a double lanechange road, the $4 \mathrm{WS}$ vehicle with yaw rate tracking control has the following advantages.

It can track the referenced sideslip angle and yaw rate, and thus improves vehicle handling stability.

It has a preferable robustness to tire cornering stiffness. The vehicle can remain stable even in emergency braking condition.

Though the resonance frequency analysis of driver/vehicle closed-loop system is still in development, it may be an effective method to study the handling stability of vehicle.

\section{ACKNOWLEDGEMENT}

This work was supported in part by the National Science Foundation of China (Grant No. 50575041).

\section{REFERENCES}

[1] Pasternack, S. (1973). Some modern control aspects of automatically steered vehicles. ASME Paper, no. 73-ICT-54.

[2] Tadahiko, T., Noritaka, Y., Shigeki, F. (1986). Improvement of vehicle dynamics by vehicle-speedsensing four-wheel steering system. SAE Paper, no. 860624, DOI:10.4271/860624.

[3] Fujita, K., Ohashi, K., Fukatani, K. (1998). Development of active rear steer system applying $\mathrm{H} \infty-\mu$ synthesis, SAE Paper, no. 981115, DOI:10.4271/981115.

[4] Pascali, L., Gabrielli, P., Caviasso, G. (2003). Improving vehicle handling and comfort performance using 4WS. SAE Paper, no. 2003-01-0961, DOI:10.4271/2003-010961.

[5] Topping, R. (2012). Understeer concepts with extensions to four-wheel steer, active steer, and time transients. SAE International Journal of Passenger Cars- Mechanical System, vol. 5, no. 1, p. 167-186, DOI:10.4271/2012-01-0245.
[6] Sano, S., Furukawa, Y., Shiralshl, S. (1986). Four wheel steering with rear wheel steer angle controlled as a function of steering wheel angle. SAE Paper, no. 860625, DOI: $10.4271 / 860625$.

[7] Marino, R., Scalzi, S. (2010). Asymptotic sideslip angle and yaw rate decoupling control in four-wheel steering vehicles. Vehicle System Dynamics, vol. 48, no. 9, p. 999-1019, DOI:10.1080/00423110903248686.

[8] Nagai, M., Hirano, Y., Yamanaka, S. (1997). Integrated control of active rear wheel steering and direct yaw moment. Vehicle System Dynamics, vol. 27, no. 5, p. 357-370, DOI:10.1080/00423119708969336.

[9] Song, J. (2012). Integrated control of brake pressure and rear-wheel steering to improve lateral stability with fuzzy logic. International Journal of Automotive Technology, vol. 13, no. 4, p. 563-570, DOI:10.1007/ s12239-012-0054-Z.

[10] Shen, X.M., Yu, F. (2008). Design and analysis of an $\mathrm{H} \infty$ integrated control system consisting of active suspension and four-wheel steering. International Journal of Vehicle Autonomous Systems, vol. 6, no. 3-4, p. 342-360, DOI:10.1504/IJVAS.2008.023591.

[11] Yin, G.D., Chen, N., Wang, J.X, Wu, L.Y. (2011). A study on $\mu$-synthesis control for four-wheel steering system to enhance vehicle lateral stability. Journal of Dynamic Systems, Measurement, and Control, vol. 133, no. 1, p. 1-6, DOI:10.1115/1.4002707.

[12] Hiraoka, T., Nishihara, O., Kumamoto, H. (2004). Model-following sliding mode control for active four-wheel steering vehicle. Review of Automotive Engineering, vol. 25, no. 3, p. 305-313.

[13] Lv, H.M., Chen, N., Li, P. (2004). Multi-objective Hळ optimal control for four-wheel steering vehicle based on yaw rate tracking. Proceedings of the Institution of Mechanical Engineers, Part D: Journal of Automobile Engineering, vol. 218, no. 10, p. 217-223, DOI:10.1177/095440700421801006.

[14] Yaniv, O. (1997). Robustness to speed of 4WS vehicles for yaw and lateral dynamics. Vehicle System Dynamics, vol. 27, no. 4, p. 221-234, DOI: $10.1080 / 00423119708969329$.

[15] Sorgatz, U. (1975). Simulation of directional behaviour of road vehicles. Vehicle System Dynamics, vol. 5, no. 1/2, p. 47-66, DOI:10.1080/00423117508968405.

[16] Weir, D., DiMarco, R.J., McRuer, D.T. (1977). Evaluation and Correlation of Driver/Vehicle Data: Summary Report. NHTSA Report STI-TR1068-1-VOL-1, National Highway Traffic Safety Administration, Washington.

[17] Bourmistrova, A., Simic, M., Hoseinnezhad, R., Jazar, R. (2011). Autodriver algorithm. Journal of Systemics, Cybernetics and Informatics, vol. 9, no. 1, p. 59-66.

[18] Canale, M., Fagiano, L. (2008). Stability control of 4WS vehicles using robust IMC techniques. Vehicle System Dynamics, vol. 46, no. 11, p. 991-1011, DOI:10.1080/00423110701790723.

[19] Genta, G. (2003). Motor Vehicle Dynamics. World Scientific, Singapore. 
[20] Nagai, M., Nishizawa, Y., Teranishi, K. (1991). Stability of $4 \mathrm{WS}$ vehicle based on side slip zeroing control: Influence of steering system dynamics. $S A E$ Paper, no. 912564, DOI:10.4271/912564.

[21] Rix, J.J., Cole, D.J. (2002). Models of human learning applicable to the vehicle steering task. $6^{\text {th }}$ International Symposium on Advanced Vehicle Control (AVEC 2002), Hiroshima, p. 561-568.

[22] Donges, E. (1978). A two-level model of driver steering behavior. Human Factors, vol. 20, no.6, p. 691-707, DOI: $10.1177 / 001872087802000607$.

[23] Guo, K.H., Guan, H. (1993). Modeling of driver / vehicle directional control system. Vehicle System Dynamics, vol. 22, no. 3, p. 141-184, DOI:10.1080/00423119308969025.
[24] Ashkenas, I.L., McRuer, D.T.(1962). A theory of handling qualities derived from pilot-vehicle system considerations. Aerospace Engineering, vol. 21, no. 2, p. $60-102$.

[25] Guo, K., Ma, F., Kong, F.(2002). Driver model parameter identification of the driver-vehicle-road closed-loop system. Automotive Engineering, vol. 24, no. 1, p. 20-24. (in Chinese)

[26] Tan, H.S., Bougler, B., Zhang, W.B. (2002). Automatic steering based on roadway markers - from highway driving to precision docking. Vehicle System Dynamics, vol. 37, no. 5, p. 315-339, DOI:10.1076/ vesd.37.5.315.3526. 